\title{
ADESÃO AO TRATAMENTO DA DOR CRÔNICA
}

\section{Estudo de variáveis demográficas, terapêuticas e psicossociais}

\author{
Geana Paula Kurita ${ }^{1}$, Cibele Andrucioli de Mattos Pimenta ${ }^{2}$
}

\begin{abstract}
RESUMO - Os objetivos deste estudo foram identificar a prevalência da adesão ao tratamento medicamentoso em pacientes com dor crônica, e analisar as relações entre adesão e características do tratamento medicamentoso e fatores psicossociais (crenças frente à dor, locus de controle da saúde e depressão). Trinta indivíduos foram avaliados 5 vezes durante 6 meses. Adesão plena ocorreu entre 43,3\% e 56,7\% dos pacientes. A adesão parcial e a não adesão foram altas (40,0\%-56,7\%). Os índices de adesão não variaram ao longo de seis meses. Menor adesão relacionou-se à ocorrência de efeitos colaterais e às crenças de que o controle da saúde dependia de si próprio, de que dor é incapacitante, de que dor indica a presença de dano físico e de que comportamentos de solicitude de outrem quando há manifestação de dor são desejáveis. Conhecer os fatores envolvidos na adesão permite testar intervenções que a otimizem.
\end{abstract}

PALAVRAS-CHAVE: dor, cooperação do paciente, atitudes, controle interno-externo/local de controle, depressão.

\begin{abstract}
Compliance with chronic pain treatment: study of demographic, therapeutic and psychosocial variables ABSTRACT - The aims of this study were to identify the prevalence of compliance with drug therapy in patients with chronic pain and analyze the relationships between compliance and characteristics of drug therapy and psychosocial factors (beliefs regarding pain, health locus of control and depression). Thirty patients were evaluated 5 times over a period of 6 months. Total compliance occurred in $43.3 \%$ to $56.7 \%$ of the patients. Partial compliance and non-compliance were high (40.0\%-56.7\%). The index of compliance did not vary over the six months. Low compliance related to occurrence of side effects and beliefs that the control of health depended on the patient, pain is a disability, that pain means the presence of physical injury and solicitous behavior of others is desirable when there is manifestation of pain. Knowing the factors involved in compliance enables us to test interventions that optimize it.
\end{abstract}

KEY WORDS: pain, patient compliance, attitudes, locus of control, depression.

Aderir ao tratamento significa aceitar a terapêutica proposta e seguí-la adequadamente. Vários fatores influenciam na adesão: características da terapia, peculiaridades do paciente, aspectos do relacionamento com a equipe multiprofissional e variáveis sócio-econômicas, entre outros ${ }^{1-5}$.

Há estudos sobre a interferência desses fatores na adesão ao tratamento de diversas doenças, porém poucos referem-se à adesão à terapia farmacológica em indivíduos com dor crônica. Indivíduos com dores crônicas, em geral, possuem longa história de dor, acentuado sofrimento psíquico, comprometimento laborativo e físico e descrença com o tratamento, por experiências anteriores nas quais os resultados foram insatisfatórios. Tais condições podem favorecer a não adesão, prolongar a dor e o sofrimento, ocasionar prejuízos à funcionalidade física e psíquica e deteriorar a qualidade de vida.

A observação clínica sugere que o número de pacientes que não aderem ao tratamento medicamentoso da dor crônica é elevado, indicando a necessidade de estudos sobre o tema. Os objetivos deste estudo foram identificar a prevalência de adesão plena, adesão parcial e não adesão ao tratamento em pacientes com dor crônica, caracterizar os motivos da adesão e buscar relações entre adesão e variáveis demográficas, psicossocioculturais e de características da dor e do tratamento.

\footnotetext{
'Enfermeira, Doutoranda da Escola de Enfermagem da Universidade de São Paulo (EE-USP), São Paulo SP, Brasil; ${ }^{2}$ Enfermeira, Professora Associada do Departamento de Enfermagem Médico-Cirúrgica da EE-USP. Estudo financiado pela Fundação de Amparo à Pesquisa do Estado de São Paulo -FAPESP.
}

Recebido 20 Março 2002, recebido na forma final 20 Novembro 2002. Aceito 2 Dezembro 2002.

Dra. Cibele A.M. Pimenta - Depto ENC; Escola de Enfermagem da USP - Avenida Dr. Enéas de Carvalho Aguiar 419 - $05403-000$ São Paulo SP Brasil. 


\section{MÉTODO}

A amostra de conveniência foi composta por 30 pacientes com dor crônica não oncológica, em tratamento no Ambulatório de Dor da Clínica Neurológica do Hospital das Clínicas da Faculdade de Medicina da Universidade de São Paulo, que foram avaliados, prospectivamente, cinco vezes ao longo de seis meses, entre os anos de 1999 e 2000. Na Figura 1 estão demonstrados os critérios de inclusão e os procedimentos de coleta de dados. $O$ estudo foi aprovado pela Comissão de Ética para Análise de Projetos de Pesquisa do Hospital das Clínicas da Faculdade de Medicina da Universidade de São Paulo e todos os pacientes assinaram o Termo de Consentimento Pós-Informação. Os dados demográficos e de caracterização da dor estão apresentados no Quadro 1.

As variáveis terapêuticas estudadas foram o número de comprimidos prescritos para serem ingeridos diariamente, o número de ingestões da medicação ao dia, razões da não adesão relatadas pelo paciente, ocorrência de efeitos colaterais e o uso de auto medicação.

As características da dor estudadas foram a intensidade, o tempo (meses) e o diagnóstico (síndrome generalizada ou localizada). A intensidade foi avaliada por meio da escala verbal de 0 a $10(0=$ ausência de dor, 1 a $3=$ dor leve, 4 a $7=$ dor moderada e 8 a $10=$ dor intensa).

As variáveis psicoculturais avaliadas foram a depressão, o locus de controle da saúde e as atitudes frente à dor.

A avaliação de sintomas depressivos foi feita por meio do Inventário de Depressão de Beck-IDB ${ }^{6}$ e categorizado em ausência de depressão, escore de 1 a 14; disforia, escore de 15 a 20; e depressão, escore acima de $20^{7}$.
O locus de Controle da Saúde (percepção das pessoas sobre a fonte de controle dos acontecimentos) foi avaliado por meio da Escala de Locus de Controle da Saúde $\mathrm{ELCS}^{8,9}$. Esse questionário permite a identificação de 3 dimensões do locus de controle: a dimensão pessoal (convicção do sujeito de controlar a sua vida), a dimensão social (outros sujeitos poderosos como fonte de controle) e a dimensão impessoal (sorte, acaso e destino como responsáveis pela situação). A partir dessas dimensões pode-se identificar dois extremos de controle: externo (outros poderosos, o acaso, a sorte e o destino) e interno (o próprio sujeito). Os escores variam entre 0 e 30 , e escores mais elevados representam crença mais intensa. $O$ índice de internalidade total é calculado por meio de uma fórmula que engloba as subescalas envolvidas na internalidade.

Para a avaliação das atitudes foi utilizado o Inventário de Atitudes frente à Dor - IAD ${ }^{10,11}$, validado para a língua portuguesa ${ }^{12}$. O IAD é composto de 7 sub-escalas, que avaliam a crença do paciente em relação a possibilidade de controle pessoal sobre a dor, relação entre emoção e intensidade de dor, dor como incapacitante, dor como indicativo de dano físico, medicamentos como o melhor tratamento para a dor crônica, solicitude de outros para a pessoa com dor e existência de cura médica para a dor crônica. A média de cada escala pode variar de 0 a 4 , sendo que o valor "desejável" para cada domínio é: controle $=4$, emoção $=4$, incapacidade $=0$, dano físico $=0$, medicação $=0$, solicitude $=0$, cura médica $=0$.

A avaliação da adesão foi feita mediante o relato do paciente e classificada em adesão plena (uso correto de todas as medicações), adesão parcial (uso de pelo menos

Quadro 1 . Características demográficas e da dor dos pacientes.

\begin{tabular}{|c|c|c|c|}
\hline Características & Amostra & Características & Amostra \\
\hline Sexo & & Renda familiar & \\
\hline masculino & $4(13,3 \%)$ & média \pm DP & $8,6 \pm 7,1$ \\
\hline feminino & $26(86,7 \%)$ & mediana & 6,0 \\
\hline Estado civil & & variação & $0-25$ \\
\hline com parceiro & $14(46,7 \%)$ & Renda per capita & \\
\hline sem parceiro & $16(53,3 \%)$ & média $\pm \mathrm{DP}$ & $3,3 \pm 2,8$ \\
\hline Idade (anos) & & mediana & 2,5 \\
\hline média \pm DP & $52,6 \pm 14,4$ & variação & $0-12,5$ \\
\hline mediana & 52,0 & Diagnóstico da dor & \\
\hline variação & $24-76$ & síndrome localizada (lombalgia, & $8(26,7 \%)$ \\
\hline Escolaridade (anos) & & sindrome miofascial e lesão por & \\
\hline média \pm DP & $6,2 \pm 4,1$ & esforços repetitivos) & \\
\hline mediana & 4,0 & síndrome generalizada & $22(73,3 \%)$ \\
\hline variação & $0-15$ & (fibromialgia e neuropatia) & \\
\hline Ocupação & & Tempo de dor em meses $\left(n=29^{*}\right)$ & \\
\hline aposentado/ licença médica & $17(60 \%)$ & média $\pm \mathrm{DP}$ & $57,3 \pm 52,5$ \\
\hline atividade remunerada & $9(30 \%)$ & mediana & 36,0 \\
\hline sem atividade remunerada & $3(10 \%)$ & variação & $6-216$ \\
\hline
\end{tabular}

* Retirado 1 paciente que teve tempo de dor de 600 meses.DP, Desvio Padrão. 


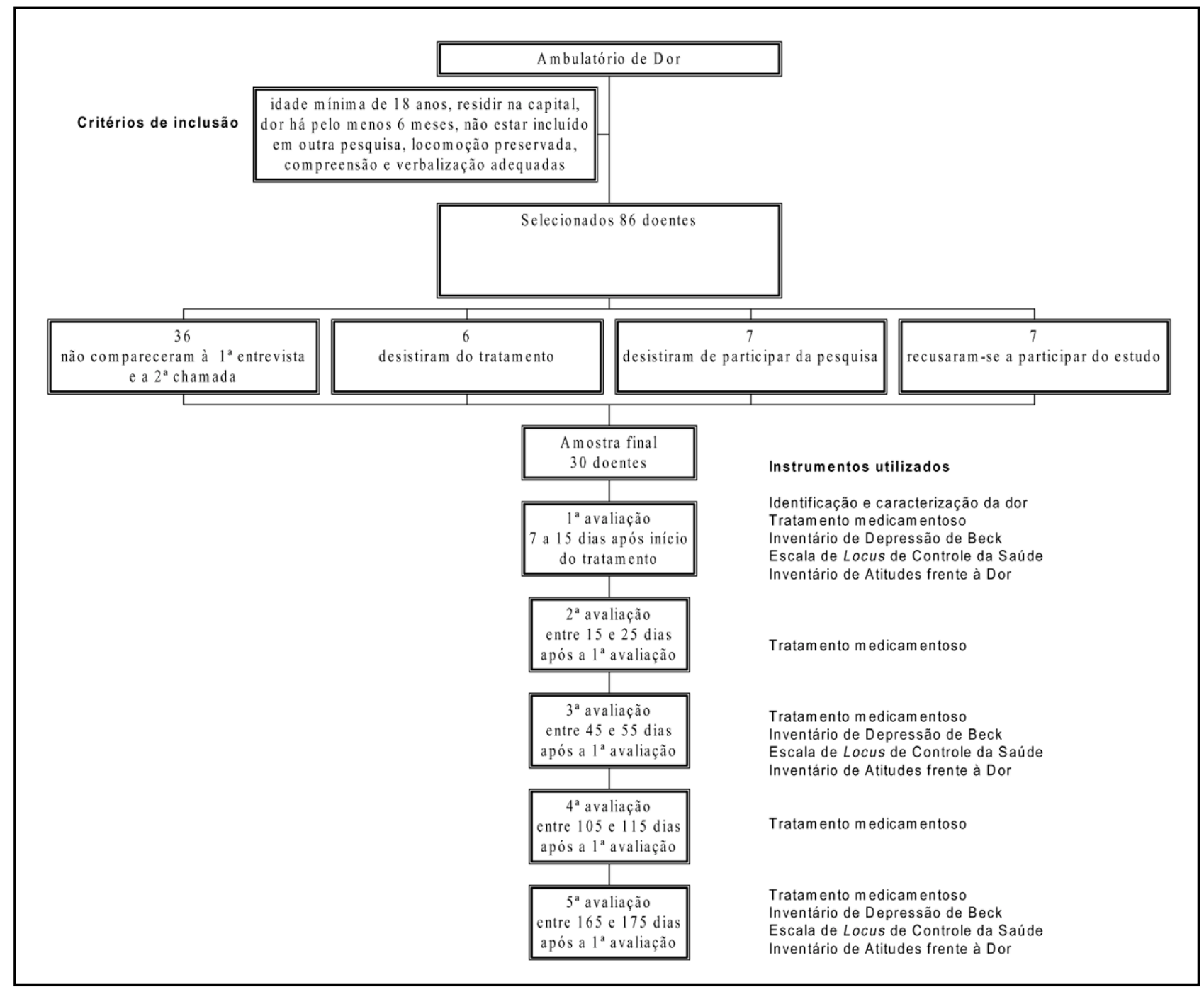

Fig 1. Fluxograma do procedimento de coleta de dados.

Tabela 1 . Distribuição dos pacientes com dor crônica de acordo com a adesão ao tratamento medicamentoso ao longo de seis meses.

\begin{tabular}{|c|c|c|c|c|c|c|c|c|c|c|c|c|}
\hline \multirow[t]{3}{*}{ Adesão } & \multicolumn{10}{|c|}{ Avaliação } & \multirow{2}{*}{\multicolumn{2}{|c|}{$\begin{array}{l}\text { Considerando } \\
\text { os } 5 \text { momentos }\end{array}$}} \\
\hline & \multicolumn{2}{|c|}{$1^{a}$} & \multicolumn{2}{|c|}{$2^{a}$} & \multicolumn{2}{|c|}{$3^{a}$} & \multicolumn{2}{|c|}{$4^{a}$} & \multicolumn{2}{|c|}{$5^{a}$} & & \\
\hline & $\mathrm{n}$ & $\%$ & $\mathrm{n}$ & $\%$ & $\mathrm{n}$ & $\%$ & $\mathrm{n}$ & $\%$ & $\mathrm{n}$ & $\%$ & $\mathrm{n}$ & $\%$ \\
\hline Plena & 13 & 43,3 & 11 & 36,7 & 14 & 46,7 & 17 & 56,7 & 14 & 46,7 & 3 & 10,0 \\
\hline Parcial & 12 & 40,0 & 14 & 46,7 & 11 & 36,7 & 7 & 23,3 & 10 & 33,3 & 27 & 90,0 \\
\hline Não & 5 & 16,7 & 5 & 16,7 & 4 & 13,3 & 5 & 16,7 & 6 & 20,0 & - & - \\
\hline Total & 30 & 100,0 & 30 & 100,0 & $29 *$ & 96,7 & $29 *$ & 96,7 & 30 & 100,0 & 30 & 100,0 \\
\hline
\end{tabular}

*Um paciente faltou na entrevista.

Tabela 2 . Estatística descritiva do Índice de Acerto de Ingestão Medicamentosa (IAIM) ao longo de seis meses.

\begin{tabular}{|c|c|c|c|c|c|c|c|c|}
\hline \multirow[t]{2}{*}{ IAIM \% } & \multicolumn{5}{|c|}{ Avaliação } & \multirow{2}{*}{$\begin{array}{l}\text { Considerando } \\
\text { os } 5 \text { momentos }\end{array}$} & \multicolumn{2}{|c|}{ Significância P } \\
\hline & $1^{a}$ & $2^{a}$ & $3^{a}$ & $4^{a}$ & $5^{a}$ & & ANOVA & Friedman \\
\hline Média & 57,2 & 58,3 & 60,3 & 69,5 & 65,6 & 62,5 & & \\
\hline Mediana & 66,7 & 66,7 & 66,7 & 100,0 & 66,7 & 63,3 & 0,587 & 0,322 \\
\hline Desvio Padrão & 43,7 & 40,3 & 43,5 & 42,3 & 39,1 & 27,6 & & \\
\hline
\end{tabular}


Tabela 3. Distribuição da referência dos pacientes sobre a ocorrência de efeitos colaterais de acordo com a adesão, ao longo de seis meses.

\begin{tabular}{|c|c|c|c|c|c|}
\hline \multirow[t]{3}{*}{ Adesão } & \multicolumn{4}{|c|}{ Efeitos colaterais } & \multirow{3}{*}{$\begin{array}{l}\text { Significância } \\
\text { (P) Teste } \\
\text { Exato de Fisher }\end{array}$} \\
\hline & \multicolumn{2}{|c|}{ Não } & \multicolumn{2}{|c|}{ Sim } & \\
\hline & $\mathrm{n}$ & $\%$ & $\mathrm{n}$ & $\%$ & \\
\hline \multicolumn{6}{|l|}{ 1a Avaliação } \\
\hline Plena & 10 & 47,6 & 3 & 33,3 & 0,691 \\
\hline Parcial/Não adesão & 11 & 52,4 & 6 & 66,7 & \\
\hline Total & 21 & 100,0 & 9 & 100,0 & \\
\hline \multicolumn{6}{|l|}{ 2ª Avaliação } \\
\hline Plena & 8 & 40,0 & 3 & 30,0 & 0,702 \\
\hline Parcial/Não adesão & 12 & 60,0 & 7 & 70,0 & \\
\hline Total & 20 & 100,0 & 10 & 100,0 & \\
\hline \multicolumn{6}{|l|}{ 3a Avaliação } \\
\hline Plena & 12 & 63,2 & 2 & 20,0 & $0,050^{*}$ \\
\hline Parcial/Não adesão & 7 & 36,8 & 8 & 80,0 & \\
\hline Total & 20 & 100,0 & 10 & 100,0 & \\
\hline \multicolumn{6}{|l|}{ 4a Avaliação } \\
\hline Plena & 16 & 72,7 & 1 & 14,3 & $0,011^{*}$ \\
\hline Parcial/Não adesão & 6 & 27,3 & 6 & 85,7 & \\
\hline Total & 23 & 100,0 & 7 & 100,0 & \\
\hline \multicolumn{6}{|l|}{ 5a Avaliação } \\
\hline Plena & 1 & 57,1 & 2 & 22,2 & 0,118 \\
\hline Parcial/Não adesão & 9 & 42,9 & 7 & 77,8 & \\
\hline Total & 21 & 100,0 & 9 & 100,0 & \\
\hline
\end{tabular}

${ }^{*} P<0,05$ - estatisticamente significativo.

uma das medicações) e não adesão (não utilização de nenhuma das medicações prescritas). Os dados sobre adesão foram analisados calculando-se o percentual de adesão plena, adesão parcial e não adesão, descrevendo-se a evolução da adesão ao longo de seis meses e determinando-se o Índice de Acerto de Ingestão Medicamentosa (IAIM). O IAIM consistiu do cálculo percentual das medicações tomadas corretamente pelo paciente no período de avaliação.

As diferenças nas frequências e sua relação com a adesão foram analisadas por meio de testes paramétricos e não paramétricos. Foram utilizados os testes t de Student, ANOVA, Mann-Whitney, Kruskall-Wallis, Fisher e Friedman. No caso das variáveis contínuas foram analisados os coeficientes de correlação de Pearson e Spearman. O nível de significância foi determinado em 5\%.

\section{RESULTADOS}

\section{Adesão ao tratamento}

Verificou-se, ao longo de seis meses, que o número de pacientes plenamente aderentes foi prevalente em quatro das cinco avaliações. No entanto, quando somados, os parcialmente aderentes e os não aderentes (variação entre $40,0 \%$ a $56,7 \%$ ) foram superiores aos plenamente aderentes em quatro das cinco avaliações. Ao final do período de seis meses 27 pacientes foram parcialmente aderentes e apenas 3 plenamente aderentes (Tabela 1). Os índices de adesão plena, parcial e não adesão permaneceram estáveis nas cinco avaliações.

O maior Índice de Acerto de Ingestão Medicamentosa (IAIM) foi de $69,5 \%$ na quarta avaliação e o menor foi de $57,2 \%$ na primeira avaliação (Tabela 2). Em todos os momentos observou-se erro na ingestão medicamentosa. Os testes estatísticos revelaram que a adesão, expressa pelo IAIM, não variou ao longo de seis meses.

\section{Relações entre adesão e características demográficas, da dor e do tratamento.}

Não foram observadas diferenças estatisticamente significativas entre os pacientes com adesão plena, adesão parcial ou não adesão e a idade, o estado civil, a escolaridade, a ocupação, a renda familiar e a renda per capita. Não foi possível analisar as relações entre adesão e sexo e ocupação, pelo pequeno número de homens (4) e de algumas ocupações.

O diagnóstico, o tempo e a intensidade de dor não mostraram relação significativa com a adesão, mas os pacientes parcialmente aderentes/não aderentes apresentaram, nas 5 avaliações, as maiores 
Quadro 2. Coeficientes de correlação obtidos na análise da relação entre o escore de Locus de Controle da Saúde e a média do Índice de Acerto de Ingestão medicamentosa (IAIM) ao final de seis meses.

\begin{tabular}{|c|c|c|c|c|}
\hline Avaliações & $\begin{array}{c}\text { Coeficiente } \\
\text { de correlação } \\
\text { de Pearson (r) }\end{array}$ & Significância (P) & $\begin{array}{c}\text { Coeficiente } \\
\text { de correlação de } \\
\text { Spearman }(r)\end{array}$ & Significância $(P)$ \\
\hline \multicolumn{5}{|c|}{ Pessoal (internalidade) } \\
\hline 1a avaliação & $-0,210$ & 0,266 & $-0,170$ & 0,368 \\
\hline 3a avaliação & $-0,425$ & $0,022^{*}$ & $-0,413$ & $0,026^{*}$ \\
\hline 5a avaliação & $-0,269$ & 0,151 & $-0,236$ & 0,209 \\
\hline \multicolumn{5}{|c|}{ Social (outros poderosos) } \\
\hline 1a avaliação & 0,063 & 0,741 & 0,119 & 0,533 \\
\hline 3a avaliação & $-0,225$ & 0,241 & $-0,205$ & 0,286 \\
\hline 5a avaliação & $-0,230$ & 0,222 & $-0,190$ & 0,314 \\
\hline \multicolumn{5}{|c|}{ Impessoal (externalidade) } \\
\hline 1a avaliação & $-0,101$ & 0,597 & $-0,126$ & 0,507 \\
\hline 3a avaliação & $-0,196$ & 0,308 & $-0,211$ & 0,271 \\
\hline 5a avaliação & $-0,053$ & 0,783 & $-0,030$ & 0,876 \\
\hline \multicolumn{5}{|c|}{ Internalidade total } \\
\hline 1a avaliação & $-0,267$ & 0,153 & $-0,283$ & 0,129 \\
\hline 3a avaliação & $-0,373$ & $0,046^{*}$ & $-0,376$ & $0,044^{*}$ \\
\hline 5a avaliação & $-0,158$ & 0,405 & $-0,122$ & 0,521 \\
\hline
\end{tabular}

${ }^{*} \mathrm{P}<0,05$ - estatisticamente significativo.

Tabela 4. Distribuição dos pacientes segundo o escore de depressão obtido pelo Inventário de Depressão de Beck e o comportamento de adesão, ao longo de seis meses.

\begin{tabular}{|c|c|c|c|c|c|}
\hline \multirow[t]{4}{*}{ Escore de depressão } & \multicolumn{4}{|c|}{ Adesão } & \multirow{4}{*}{$\begin{array}{l}\text { Significância } \\
\text { (P) Teste } \\
\text { Exato } \\
\text { de Fisher }\end{array}$} \\
\hline & \multirow{2}{*}{\multicolumn{2}{|c|}{ Plena }} & \multirow{2}{*}{\multicolumn{2}{|c|}{$\begin{array}{c}\text { Parcial/ } \\
\text { Não adesão }\end{array}$}} & \\
\hline & & & & & \\
\hline & $\mathrm{n}$ & $\%$ & $\mathrm{n}$ & $\%$ & \\
\hline \multicolumn{6}{|l|}{ 1’a avaliação } \\
\hline Escore até 20 & 12 & 92,3 & 14 & 82,4 & \\
\hline Escore acima de 20 & 1 & 7,7 & 3 & 17,6 & 0,613 \\
\hline $\mathrm{N}$ de pacientes & 13 & 100,0 & 17 & 100,0 & \\
\hline \multicolumn{6}{|l|}{ 3a avaliação } \\
\hline Escore até 20 & 12 & 85,7 & 13 & 86,7 & \\
\hline Escore acima de 20 & 2 & 14,3 & 2 & 13,3 & 1,000 \\
\hline $\mathrm{N}$ de pacientes & 14 & 100,0 & 15 & 100,0 & \\
\hline \multicolumn{6}{|l|}{ 5a avaliação } \\
\hline Escore até 20 & 11 & 78,6 & 14 & 87,5 & \\
\hline Escore acima de 20 & 3 & 21,4 & 2 & 12,5 & 0,642 \\
\hline $\mathrm{N}$ de pacientes & 14 & 100,0 & 16 & 100,0 & \\
\hline
\end{tabular}

médias de intensidade de dor. A dor diminuiu ao longo do tratamento, comparando-se a primeira avaliação (média $=8,3$ ) com as demais ( $p=0,008$, ANOVA e $p=0,003$, Friedman). A menor média de intensidade da dor foi 6,5 , na quarta avaliação.

Relações entre adesão e variáveis terapêuticas

Os fármacos prescritos (antiinflamatórios, analgésicos, antidepressivos, neurolépticos e anticonvul- sivantes) foram todos de uso oral. A razão mais citada para adesão plena foi "o remédio melhora a dor"(54,0\% a 63,9\%). Para a adesão parcial foi citada "sente-se mal, tem efeitos colaterais" $(55,6 \%$ a $66,6 \%$ ), e para a não adesão "indisponibilidade na farmácia do hospital" (23,1\% a $28,9 \%)$, "sente-se mal, tem efeitos colaterais" (25\%) e "dinheiro insuficiente para a compra" (15,4\% a $23,7 \%)$.

A média do número de comprimidos ingeridos 
Tabela 5. Distribuição dos pacientes de acordo com a adesão e a média de escore dos domínios do Inventário de Atitudes frente à Dor (IAD), ao longo de seis meses.

\begin{tabular}{|c|c|c|c|c|}
\hline \multirow[t]{2}{*}{ Avaliação } & \multicolumn{2}{|c|}{ Adesão } & \multicolumn{2}{|c|}{ Significância (P) } \\
\hline & Plena & Parcial/Não adesão & T Student & Mann-Whitney \\
\hline \multicolumn{5}{|l|}{ Controle } \\
\hline 1ạ Média \pm DP & $2,1 \pm 1,2$ & $1,7 \pm 1,0$ & 0,370 & 0,367 \\
\hline 3a Média \pm DP & $2,1 \pm 1,2$ & $2,0 \pm 1,1$ & 0,969 & 0,930 \\
\hline 5 a Média \pm DP & $2,2 \pm 1,3$ & $2,0 \pm 0,9$ & 0,621 & 0,631 \\
\hline \multicolumn{5}{|l|}{ Emoção } \\
\hline 1a Média $\pm D P$ & $2,5 \pm 1,4$ & $1,9 \pm 1,3$ & 0,205 & 0,152 \\
\hline 3a Média \pm DP & $2,6 \pm 1,4$ & $2,3 \pm 1,5$ & 0,516 & 0,523 \\
\hline 5 a Média $\pm D P$ & $2,6 \pm 1,4$ & $2,0 \pm 1,5$ & 0,298 & 0,425 \\
\hline \multicolumn{5}{|l|}{ Incapacidade } \\
\hline 1 a Média $\pm \mathrm{DP}$ & $2,6 \pm 1,3$ & $2,7 \pm 1,2$ & 0,696 & 0,717 \\
\hline 3a Média $\pm D P$ & $2,4 \pm 1,4$ & $2,5 \pm 1,4$ & 0,875 & 0,876 \\
\hline 5 a Média $\pm D P$ & $1,8 \pm 1,4$ & $2,9 \pm 1,1$ & $0,021^{*}$ & $0,023^{*}$ \\
\hline \multicolumn{5}{|l|}{ Dano físico } \\
\hline 1 a Média $\pm \mathrm{DP}$ & $2,5 \pm 1,2$ & $1,9 \pm 1,5$ & 0,257 & 0,247 \\
\hline 3a Média \pm DP & $2,1 \pm 1,2$ & $2,3 \pm 1,4$ & 0,627 & 0,582 \\
\hline 5 a Média $\pm \mathrm{DP}$ & $1,7 \pm 1,3$ & $2,8 \pm 1,2$ & $0,021^{*}$ & $0,031 *$ \\
\hline \multicolumn{5}{|l|}{ Medicação } \\
\hline 1a Média \pm DP & $2,8 \pm 1,1$ & $2,6 \pm 1,2$ & 0,528 & 0,498 \\
\hline 3a Média \pm DP & $2,9 \pm 1,3$ & $2,9 \pm 1,0$ & 0,993 & 0,723 \\
\hline 5a Média \pm DP & $3,0 \pm 1,0$ & $3,0 \pm 1,2$ & 0,984 & 0,782 \\
\hline \multicolumn{5}{|l|}{ Solicitude } \\
\hline 1 a Média \pm DP & $1,6 \pm 1,3$ & $1,7 \pm 1,3$ & 0,768 & 0,720 \\
\hline 3a Média \pm DP & $1,3 \pm 1,5$ & $1,8 \pm 1,2$ & 0,300 & 0,147 \\
\hline 5a Média \pm DP & $1,4 \pm 1,6$ & $2,1 \pm 1,1$ & 0,142 & 0,094 \\
\hline \multicolumn{5}{|l|}{ Cura médica } \\
\hline 1a Média \pm DP & $2,7 \pm 0,5$ & $2,8 \pm 0,6$ & 0,811 & 0,673 \\
\hline 3a Média \pm DP & $2,7 \pm 0,6$ & $2,6 \pm 0,8$ & 0,781 & 0,894 \\
\hline 5 a Média \pm DP & $2,4 \pm 0,9$ & $2,4 \pm 0,9$ & 0,939 & 0,848 \\
\hline
\end{tabular}

foi alta, 5 comprimidos/dia, assim como a média do número de tomadas de medicação, 4 ou mais vezes/ dia. Não se encontrou diferença significativa entre essas variáveis e a adesão.

Durante os seis meses de avaliação houve 45 relatos de efeitos colaterais e, em $86,7 \%$ das vezes, houve referência a um ou dois efeitos colaterais. $O$ número de pacientes sem efeitos colaterais foi pelo menos duas vezes maior que o dos que relataram efeitos colaterais, nas cinco avaliações. Do total de queixas manifestadas, $77,7 \%$ foram de pacientes que aderiram parcialmente ou não aderiram ao tratamento. Na terceira e quarta avaliações houve associação entre efeito colateral e adesão (Tabela 3).

Da análise sobre o uso de automedicação para controle da dor e adesão não se encontrou relação estatisticamente significativa. Nas 5 avaliações, en- tre 1/3 e 1/2 dos pacientes utilizava algum remédio por conta própria. As medicações citadas incluíram analgésico, benzodiazepínico e antibiótico.

\section{Relação entre adesão e variáveis psicossocioculturais}

Os testes estatísticos demonstraram que entre as variáveis psicossocioculturais (depressão, locus de controle e atitudes), apenas a atitude frente à cura médica variou ao longo do tempo $(p=0,021)$, indicando que a crença na cura médica diminuiu ao longo do tratamento.

A média do escore de depressão, nas três avaliações, foi em torno de 14 (compatível com ausência de depressão). Escores acima de 15 (disforia ou depressão) foram observados em parcela significativa dos pacientes na primeira $(46,7 \%)$, terceira $(34,5 \%)$ 
Quadro 3. Coeficientes de correlação resultantes da análise entre o escore obtido nos domínios do Inventário de Atitudes frente à Dor e a média do Índice de Acerto de Ingestão Medicamentosa, ao final de seis meses.

\begin{tabular}{|c|c|c|c|c|}
\hline Avaliações & $\begin{array}{l}\text { Coeficiente } \\
\text { de correlação } \\
\text { de Pearson (r) }\end{array}$ & Significância (P) & $\begin{array}{c}\text { Coeficiente } \\
\text { de correlação de } \\
\text { Spearman }(r)\end{array}$ & Significância (P) \\
\hline \multicolumn{5}{|l|}{ Controle } \\
\hline 1ạ avaliação & $-0,103$ & 0,588 & $-0,143$ & 0,450 \\
\hline 3a avaliação & $-0,063$ & 0,744 & $-0,138$ & 0,476 \\
\hline 5’a avaliação & $-0,139$ & 0,462 & $-0,169$ & 0,373 \\
\hline \multicolumn{5}{|l|}{ Emoção } \\
\hline 1a avaliação & $-0,003$ & 0,987 & 0,029 & 0,881 \\
\hline 3a avaliação & 0,087 & 0,652 & 0,124 & 0,520 \\
\hline 5’a avaliação & 0,208 & 0,269 & 0,184 & 0,332 \\
\hline \multicolumn{5}{|l|}{ Incapacidade } \\
\hline 1a avaliação & $-0,225$ & 0,231 & $-0,182$ & 0,336 \\
\hline 3a avaliação & $-0,115$ & 0,553 & $-0,123$ & 0,526 \\
\hline 5a avaliação & $-0,052$ & 0,787 & $-0,040$ & 0,836 \\
\hline \multicolumn{5}{|l|}{ Dano físico } \\
\hline 1a avaliação & $-0,002$ & 0,994 & 0,002 & 0,992 \\
\hline 3aa avaliação & $-0,143$ & 0,458 & $-0,112$ & 0,562 \\
\hline $5^{a}$ avaliação & $-0,317$ & 0,088 & $-0,304$ & 0,103 \\
\hline \multicolumn{5}{|l|}{ Medicação } \\
\hline 1a avaliação & 0,089 & 0,640 & 0,063 & 0,741 \\
\hline 3’a avaliação & $-0,245$ & 0,200 & $-0,200$ & 0,297 \\
\hline 5a avaliação & $-0,144$ & 0,448 & $-0,130$ & 0,494 \\
\hline \multicolumn{5}{|l|}{ Solicitude } \\
\hline 1a avaliação & $-0,159$ & 0,400 & $-0,208$ & 0,270 \\
\hline 3ª avaliação & $-0,336$ & 0,075 & $-0,403$ & $0,030 *$ \\
\hline 5a avaliação & $-0,268$ & 0,152 & $-0,288$ & 0,123 \\
\hline \multicolumn{5}{|l|}{ Cura médica } \\
\hline 1’a avaliação & 0,028 & 0,885 & $-0,023$ & 0,903 \\
\hline 3ạ avaliação & 0,184 & 0,338 & 0,057 & 0,769 \\
\hline 5a avaliação & 0,299 & 0,109 & 0,271 & 0,147 \\
\hline
\end{tabular}

${ }^{*} \mathrm{P}<0,05$ - estatisticamente significativo.

e na quinta entrevistas (30,0\%). As relações entre adesão e depressão não se mostraram estatisticamente significativas (Tabela 4).

Quanto ao locus de controle da saúde, observouse que a maior média pertenceu à subescala Outros Poderosos, revelando que os pacientes possuíam crença maior em fontes externas de poder. Os pacientes plenamente aderentes e os parcialmente/não aderentes não apresentaram diferenças significativas nas médias das três subescala em todas as avaliações. Porém, houve correlação negativa entre o IAIM e a subescala Internalidade para a Saúde e o índice de Internalidade Total (Quadro 2). Tal relação significou que quanto maior a crença do paciente de que o controle de sua saúde depende de si próprio, menor foi o IAIM.

No estudo das atitudes frente à dor, apenas os domínios emoção e solicitude tiveram orientação desejável, apesar de distantes do "ideal". Na avaliação das relações entre Atitudes frente à Dor e adesão observou-se que os domínios incapacidade e dano físico influíram na adesão (Tabela 5). Observou-se que quanto maior a crença na incapacidade e dano físico, menor foi a adesão. A análise entre as Atitudes frente à Dor e o Índice de Acerto de Ingestão Medicamentosa demonstrou correlação negativa entre o domínio solicitude e o IAIM (Quadro 3 $p=0,030$ ), isto é, quanto maior a crença na solicitude, menor a adesão.

\section{DISCUSSÃO}

Observou-se que a adesão ao tratamento foi insuficiente e não variou ao longo do tempo, o que era 
esperado, sugerindo que dependeu de características pessoais mais do que de fatores externos. Os índices de não adesão e de acerto de ingestão observados assemelharam-se aos encontrados em outros trabaIhos ${ }^{13,14}$ e foram preocupantes, indicando a necessidade de intervenções que melhorem a adesão.

Não foram observadas diferenças entre adesão e idade, estado civil, escolaridade, ocupação e renda, à semelhança de outros estudos com pacientes com dor crônica ${ }^{3,14}$. Cabe ressaltar que a maior parte dos remédios foi gratuita.

O diagnóstico e o tempo de dor não mostraram relação significativa com a adesão. Berndt, Maier e Schütz $^{13}$ (1993) também não encontraram relação entre o local e a intensidade da dor e a adesão ao tratamento. Acreditava-se que pacientes com dores generalizadas e maior tempo de dor teriam maior aderência à terapia, o que não foi observado.

A intensidade da dor diminuiu ao longo do tratamento (na primeira avaliação foi 8,3 e a menor média foi 6,5$)$. Apesar de estatisticamente diferentes é possível que a expressão clínica da diferença foi modesta, e que isto tenha contribuído para a manutenção dos altos índices de não adesão e adesão parcial. Estudos com doenças crônicas apontam que a melhora do quadro sintomático indica adesão ao tratamento. Em dores crônicas é difícil avaliar adesão pela melhora da dor. Os quadros são acompanhados de melhora e piora, o ajuste da terapêutica leva algum tempo e nem sempre adequado controle é alcançado.

Observou-se média elevada do número de comprimidos ingeridos ao dia (5) e do número de tomadas diárias (4), que não se relacionaram com a adesão. A complexidade da terapia, o número de comprimidos e o número de tomadas diárias são citados constantemente na literatura como fatores de interferência na adesão ${ }^{15-18}$, o que não se observou no presente estudo. É possível que a homogeneidade da amostra quanto ao número de comprimidos e número de tomadas não tenha permitido a expressão de diferenças quanto à adesão. Estudos sobre efetividade relatam maior adesão aos medicamentos de dose diária única ou de liberação prolongada. Tais estudos, geralmente, avaliam uma única medicação, o que não é comum no manejo da dor crônica. Possivelmente os resultados de adesão à dose única não possam ser plenamente transpostos para esquemas mais complexos ${ }^{4}$.

Na presente pesquisa observou-se relação entre a ocorrência de efeitos colaterais e adesão. A relação entre efeitos colaterais e adesão é complexa. É possível que os efeitos desagradáveis desestimulem a continuidade da terapia. É possível também que o paciente que não adere justifique seu comportamento relatando maior número de efeitos colaterais. Efeitos colaterais desagradáveis são problemas à adesão ao tratamento e citados por vários pesquisadores $^{14,19}$, embora nem sempre explorados de modo sistemático nos estudos. O esclarecimento de que efeitos colaterais são possíveis, a atenção sistemática visando ao seu controle e enfatizar que o tratamento é estratégia importante para o controle da dor, talvez minimizem o impacto sobre a adesão.

A automedicação ocorreu em pelo menos um terço dos pacientes nas cinco avaliações. Nas dores crônicas, a possibilidade de o paciente ter prescrição para o uso de doses de "resgate" é desejável, visto as repercussões nas atividades diárias e sofrimento quando há exacerbação da dor. Na presente pesquisa, poucos pacientes $(6,7 \%)$ tinham medicação "se necessária", o que talvez os tenha levado a consumir analgésicos por conta própria.

Imaginava-se que o comportamento de adesão poderia estar associado com depressão, o que não foi observado e causou surpresa. Também chamou atenção o fato de os índices de depressão não terem se modificado ao longo de seis meses de tratamento, considerando-se que cerca de $30 \%$ dos pacientes apresentaram alteração do humor. É comum o pré-julgamento de que os pacientes não aderentes são menos colaborativos por possuírem maiores alterações psíquicas e emocionais ${ }^{3}$. No entanto, há registros na literatura de que pacientes com maiores escores em escalas que avaliaram alteração de humor tinham maior disposição para melhorar a dor e o humor ${ }^{20,21}$. Poucos estudos avaliaram a influência do estado emocional na adesão ao tratamento da dor crônica, apesar do consenso sobre a coexistência de dor e depressão $22-24$. Talvez a persistência de sintomas depressivos em parcela significativa dos pacientes tenha contribuído para o modesto alívio da dor que foi observado.

A percepção dos pacientes sobre a fonte de controle dos acontecimentos em sua saúde (locus de controle) propõe que a crença do indivíduo determina a ação a ser tomada. Quem acredita que os resultados, ao menos em parte, são dependentes de suas ações é considerado internamente orientado. Aquele que tem orientação externa acredita menos na relação entre ação individual e resultado, e enfatiza forças externas como o destino, a religião, a sorte e outros poderosos. A orientação do locus 
de controle não é fixa, e o indivíduo pode possuir diversas orientações em diferentes situações ou estágios. Conhecer a orientação do locus de controle do paciente é importante para que se antecipem as mudanças que o paciente necessitará fazer tendo em vista o melhor manejo do tratamento ${ }^{25,26}$. Na presente pesquisa observou-se correlação negativa entre a subescala Internalidade para a Saúde, o índice de Internalidade Total e o IAIM. Isto é, quanto maior a crença do paciente de que o controle de sua saúde dependia de si próprio, menor foi a adesão. Esse dado causou certa surpresa, pois tinha-se a hipótese de que maior internalidade estaria relacionada à maior adesão. No entanto, considerando-se que o alívio obtido foi modesto (melhor média de intensidade de dor foi de 6,5 ) e que o tratamento foi prolongado ( 6 meses), pode-se pensar que o paciente com orientação mais interna avaliaria a situação e tomaria a decisão de interromper a terapia, o que representou menor adesão. Talvez pacientes com orientação mais externa sejam mais obedientes, independentes da avaliação particular da situação. Há controvérsias sobre qual a orientação desejável do locus de controle nas questões de saúde 9 .

O modelo cognitivo comportamental considera que os valores, as atitudes e as crenças, participam da experiência dolorosa, influenciam na percepção, expressão e manejo da dor ${ }^{12,27}$, e há indícios de que possam influir na adesão ao plano terapêutico ${ }^{27}$.

No presente estudo, apenas os domínios emoção e solicitude tiveram escores próximos do desejável. A emoção faz parte da experiência dolorosa e a compreensão desta relação pelos pacientes pode auxiliá-los no manejo da dor. Solicitude é um comportamento cuidadoso, atencioso, que faz parte das relações humanas, sendo saudável dentro de certos limites. Porém, quando inadequado, pode estimular a dependência e perpetuar comportamentos de dor indesejáveis, mantendo a incapacidade ${ }^{12}$. Pimenta ${ }^{24}$ (1999) estudou pacientes com dor crônica e observou orientação desejável para os domínios emoção e solicitude, à semelhança do observado nesta pesquisa.

Neste estudo, os domínios incapacidade e dano físico influíram na adesão. Nos dois casos as maiores médias pertenceram a pacientes com adesão parcial ou não aderentes. Tais dados indicaram que os pacientes com menor adesão acreditavam-se mais incapacitados pela dor e que a dor indicava a existência de dano físico. É possível que os pacientes com dor crônica que se consideram fisicamente limitados pela dor, sintam-se impossibilitados de te- rem comportamentos mais ativos, o que dificultaria o seguimento do tratamento. Talvez quem se considere incapacitado pense que não há mais nada a fazer, a não ser conviver com a incapacidade. A crença de que há dano físico na existência da dor crônica é frequente entre os pacientes, embora muitas vezes isso não seja real. Há na literatura descrições de pacientes que acreditam ser portadores de lesão física e interrompem a prática de atividade física por acreditar que esta piora a lesão, e que necessita de repouso ${ }^{28-30}$. Não se encontraram estudos sobre a interrupção do uso de medicamentos e a crença de que dor indica lesão.

No presente estudo observou-se correlação negativa entre solicitude o o IAIM, isto é, pacientes com menor crença de que comportamentos de solicitude dos outros frente à sua expressão de dor são desejáveis foram os mais aderentes. Tal fato talvez indique que pacientes que necessitem menos da confirmação de "coitadinhos" sejam mais ativos, busquem a melhora "de fato", e isso os direciona a serem mais aderentes.

\section{CONCLUSÃO}

A adesão ao tratamento foi baixa. As variáveis que em algum momento se relacionaram à adesão foram: presença de efeitos colaterais, estilo de locus de controle da saúde, na dimensão Internalidade para a Saúde e índice de Internalidade Total, e as crenças frente à dor, nos domínios incapacidade, dano físico e solicitude. Tais dados são muito importantes pois mostram a influência dos aspectos cognitivos no modo de o paciente lidar com o tratamento. Apesar de essa relação ser bastante comentada na literatura, há poucos dados de pesquisa sobre ela. Conhecer os fatores envolvidos na adesão ao tratamento da dor crônica permite testar intervenções que otimizem a adesão e, talvez, melhorem o controle da dor crônica.

\section{REFERÊNCIAS}

1. Rapley P. Self-care: re-thinking the role of compliance. Austral J Adv Nursing 1997;15:21-25.

2. Hays RD, Kravitz RL, Mazel RM, et al. The impact of patient adherence on health outcomes for patients with chronic disease in the medical outcomes study. J Behav Med 1994;17:347-360.

3. King AS, Snow BR. Factors for predicting premature termination from a multidisciplinary inpatient chronic pain program. Pain 1989;39:281-287.

4. Oseasohn C, Graveley EA, Hudepohl NC. Issues in medication compliance research. Can J Nurs Res 1989;21:35-43.

5. Morisson, R. Medication noncompliance. The Can Nurs 1993;241:15-18.

6. Beck, AT. Terapia cognitiva da depressão. Rio de Janeiro: Zahar, 1982. Apêndice: materiais:385-387.

7. Gorenstein L, Andrade L. Inventário de depressão de Beck: propriedades psicométricas da versão em português. Rev Psiquiatria Clín 1998;25:245-250. 
8. Dela Coleta JA, Dela Coleta MF. Escalas para medida de atitudes e outras variáveis psicossociais. Ribeirão Preto: Escola de Enfermagem da Universidade de São Paulo, 1996 (comunicação pessoal).

9. Wallston KA, Wallston BS, DeVellis R. Development of the Multidimensional Health Locus of Control (MHLC) Scales. Health Educ Monographs 1978;6:160-170.

10. Jensen MP, Karoly P, Huger R. The development and preliminary validation of an instrument to assess patient's attitudes toward pain. J Psychosomatic Res 1987;31:393-400.

11. Tait RC, Chibnall JT. Development of a brief version of the survey of pain attitudes. Pain 1997;70:229-235.

12. Pimenta CAM. Atitudes de pacientes com dor crônica frente à dor. Tese. São Paulo: Escola de Enfermagem da USP, 1999.

13. BerndtS, Maier C, Schütz HW. Polymedication and medication compliance in patients with chronic non-malignant pain. Pain 1993;52:331-339.

14. Packard RC, $\mathrm{O}^{\prime}$ Connell P. Medication compliance among headache patients. Headache 1986;26:416-419.

15. Pierin AMG. A pessoa com hipertensão arterial em tratamento no ambulatório: estudo sobre problemas, dificuldades e expectativas quanto a doença e tratamento. Dissertação. São Paulo: Escola de Enfermagem da USP, 1985.

16. Martins JMC. A não adesão dos pacientes reumáticos ao tratamento. Rev Bras Reumatol 1987;27:215-216.

17. Insull, $\mathrm{W}$. The problem of compliance to cholesterol altering therapy. J Internal Med 1997;241:317-325.

18. Niemeyer MG, Kleinjans HAJ, Ree R de, Zwinderman AH, Cleophas TJM, Van der Wall EE. Comparison of multiple - dose and once - daily nitrate therapy in 1212 patients with stable angina pectoris: effects on quality of life indices. Angiology 1997;48:855-862.
19. Woodroffe MA, Hays H. Fentanyl transdermal system: pain management at home. Can Fam Physician 1997;43:268-272.

20. Kleinke CL, Splangler AS. Predicting treatment outcome of chronic back pain patients in a multidisciplinary pain clinic: methodological issues and treatment implications. Pain 1988;33:41-48.

21. Dolce JJ, Crocker MF, Doleys DM. Prediction of outcome among chronic pain patients. Behav Res Ther 1986;24:313-319.

22. Merskey H. Chronic pain and psychiatric illness. In Bonica J(ed.). The management of pain. 2. Philadelphia: Lea \& Febger; 1990:320-327.

23. Craig KD. Emotional aspects of pain. In Wall PD, Melzack R (eds). Textbook of pain. 3. [CD-ROM] New York: Churchill Livingstone; 1997.

24. Pimenta CAM. Aspectos culturais, afetivos e terapêuticos relacionados à dor no câncer. Tese. São Paulo: Escola de Enfermagem da USP; 1995.

25. Schroeder PS, Miller JF. Qualitative study of locus of control in patients with peripheral vascular disease. In Miller JF (ed.). Coping with chronic illness: overcoming powerlessness. Philadelphia: FA Davis, 1983:149-161.

26. Kolton KA, Piccolo P. Patient compliance: a challenge in practice. Nurse Pract 1988;13:37-50.

27. Turk DC, Meichenbaum D, Genest M. Pain and behavioral medicine: a cognitive behavioral perspective. New York: Guilford, 1983.

28. Jensen MP, Turner JA, Romano JM, Lawler BK. Relationship of pain: specific beliefs to chronic pain adjustment. Pain 1994;57:301-309.

29. Rainville J, Sobel J, Hartigan C, Monlux G, Bean J. Decreasing disability in chronic back pain through aggressive spine rehabilitation. J Rehabil Res Dev 1997;34:383-393.

30. Ruzicka AS. Pain beliefs: what do elders believe? J Holis Nurs 1998;16:369-382. 